\title{
Polynilpotent Multipliers of Some Nilpotent Products of Cyclic Groups
}

\author{
Azam Hokmabadi, Behrooz Mashayekhy, ${ }^{*}$ \\ and Fahimeh Mohammadzadeh
}

\begin{abstract}
In this article, we present an explicit formula for the $c$ th nilpotent multiplier (the Baer invariant with respect to the variety of nilpotent groups of class at most $c \geq 1$ ) of the $n$th nilpotent product of some cyclic groups $G=\mathbb{Z}^{n} \cdots * *^{n} \mathbb{Z}^{n} \mathbb{Z}_{r_{1}}{ }^{*} \cdots * \mathbb{Z}_{r_{t}}$, (m-copies of $\mathbb{Z}$ ), where $r_{i+1} \mid r_{i}$ for $1 \leq i \leq t-1$ and $c \geq n$ such that $\left(p, r_{1}\right)=1$ for all primes $p$ less than or equal to $n$. Also, we compute the polynilpotent multiplier of the group $G$ with respect to the polynilpotent variety $\mathcal{N}_{c_{1}, c_{2}, \ldots, c_{t}}$, where $c_{1} \geq n$.
\end{abstract}

2010 Mathematics Subject Classification. 20E10, 20E34, 20F12, $20 \mathrm{~F} 18$.

Key words and phrases. Polynilpotent multiplier, nilpotent product, cyclic group.

*This research was in part supported by a grant from IPM (No. 85200037). 


\section{Introduction and Motivation}

Let $G$ be any group with a free presentation $G \cong F / R$, where $F$ is a free group. Then the Baer invariant of $G$ with respect to the variety of groups $\mathcal{V}$, denoted by $\mathcal{V} M(G)$, is defined to be

$$
\mathcal{V} M(G)=\frac{R \cap V(F)}{\left[R V^{*} F\right]},
$$

where $V$ is the set of laws of the variety $\mathcal{V}, V(F)$ is the verbal subgroup of $F$ and

$$
\begin{array}{r}
{\left[R V^{*} F\right]=\left\langle v\left(f_{1}, \ldots, f_{i-1}, f_{i} r, f_{i+1}, \ldots, f_{k}\right) v\left(f_{1}, \ldots, f_{i}, \ldots, f_{k}\right)^{-1}\right|} \\
\left.r \in R, f_{i} \in F, v \in V, 1 \leq i \leq k, k \in \mathbb{N}\right\rangle .
\end{array}
$$

For example, if $\mathcal{V}$ is the variety of abelian groups $\mathcal{A}$, then the Baer invariant of the group $G$ will be $\left(R \cap F^{\prime}\right) /[R, F]$, which is isomorphic to $M(G)$, the Schur multiplier of $G$ (see [5]). If $\mathcal{V}$ is the variety of polynilpotent groups of class row $\left(c_{1}, \ldots, c_{t}\right), \mathcal{N}_{c_{1}, c_{2}, \ldots, c_{t}}$, then the Baer invariant of a group $G$ with respect to this variety, which we call a polynilpotent multiplier, is as follows:

$\mathcal{N}_{c_{1}, c_{2}, \ldots, c_{t}} M(G)=\frac{R \cap \gamma_{c_{t}+1} \circ \ldots \circ \gamma_{c_{1}+1}(F)}{\left[R,{ }_{c_{1}} F, c_{c_{2}} \gamma_{c_{1}+1}(F), \ldots, c_{t} \gamma_{c_{t-1}+1} \circ \ldots \circ \gamma_{c_{1}+1}(F)\right]}$,

where $\gamma_{c_{t}+1} \circ \cdots \circ \gamma_{c_{1}+1}(F)=\gamma_{c_{t}+1}\left(\gamma_{c_{t-1}+1}\left(\cdots\left(\gamma_{c_{1}+1}(F)\right) \cdots\right)\right)$ is the group which is attained from the iterated terms of the lower central series of $F$. See [4] for the equality

$$
\left[R \mathcal{N}^{*}{ }_{c_{1}, c_{2}, \ldots, c_{t}} F\right]=\left[R,{ }_{c_{1}} F, c_{2} \gamma_{c_{1}+1}(F), \ldots,{ }_{c_{t}} \gamma_{c_{t-1}+1} \circ \ldots \circ \gamma_{c_{1}+1}(F)\right] .
$$

Note that the Baer invariant of $G$ is always abelian and independent of the choice of the free presentation for $G$ with respect to a variety $\mathcal{V}$ (see [5]). In particular, if $t=1$ and $c_{1}=c$, then the Baer invariant of $G$ with respect to the variety $\mathcal{N}_{c}$ is called the $c$-nilpotent multiplier and given by

$$
\mathcal{N}_{c} M(G)=\frac{R \cap \gamma_{c+1}(F)}{\left[R,{ }_{c} F\right]} .
$$


Determining these Baer invariants of groups is known to be very useful for classification of groups into isologism classes with respect to the chosen varieties (see [5]). In 1979, Moghaddam [8] gave a formula for the $c$-nilpotent multiplier of a direct product of two groups, where $c+1$ is a prime number or 4. Also, in 1998, Ellis [1] presented the formula for all $c \geq 1$. In 1997, Moghaddam and Mashayekhy [7] presented an explicit formula for the $c$ nilpotent multiplier of a finite abelian group for every $c \geq 1$.

It is known that the nilpotent product is a generalization of the direct product. In 1992, Gupta and Moghaddam [2] calculated the $c$-nilpotent multiplier of the nilpotent dihedral group of class $n, G_{n}=\left\langle x, y \mid x^{2}, y^{2},[x, y]^{2 n-1}\right\rangle$. It is routine to verify that $G_{n} \cong \mathbb{Z}_{2} * \mathbb{Z}_{2}$. In 2003, Moghaddam, Mashayekhy, and Kayvanfar [9] extended the previous result and calculated the $c$-nilpotent multiplier of $n$th nilpotent products of two cyclic groups for $n=2,3$ and 4 under some conditions. Also, the second author [6] gave an implicit formula for the $c$-nilpotent multiplier of a nilpotent product of cyclic groups.

In this paper, we first obtain an explicit formula for the $c$-nilpotent multiplier of the $n$th nilpotent product of some cyclic groups $G=\underbrace{\mathbb{Z}^{n} \cdots * \mathbb{Z}^{n}}_{m-\text { copies }} *$ $\mathbb{Z}_{r_{1}} \stackrel{n}{*} \cdots \stackrel{n}{*} \mathbb{Z}_{r_{t}}$, where $r_{i+1} \mid r_{i}$ for $1 \leq i \leq t-1$ and $c \geq n$ such that $\left(p, r_{1}\right)=1$ for all primes $p$ less than or equal to $n$. This result extends the works of Moghaddam and Mashayekhy [7] and Moghaddam, Mashayekhy and Kayvanfar [9]. Second, we present an explicit formula for the polynilpotent multiplier of such a group $G$ with respect to the polynilpotent variety $\mathcal{N}_{c_{1}, c_{2}, \ldots, c_{t}}$, where $c_{1} \geq n$.

\section{Notation and Preliminaries}

Definition 2.1. ([3, $\S 11.1$ and $\S 12.3])$. The basic commutators on the letters $x_{1}, x_{2}, \ldots, x_{n}, \ldots$ are defined as follows: 
(i) The letters $x_{1}, x_{2}, \ldots, x_{n}, \ldots$ are basic commutators of weight one, ordered by setting $x_{i}<x_{j}$, if $i<j$.

(ii) Having defined the basic commutators of weight less than $n$, the basic commutators of weight $n$ are defined as $c_{k}=\left[c_{i}, c_{j}\right]$, where

(a) $c_{i}$ and $c_{j}$ are basic commutators and $w\left(c_{i}\right)+w\left(c_{j}\right)=n$, where $w(c)$ is the weight of $c$ and

(b) $c_{i}>c_{j}$, and if $c_{i}=\left[c_{s}, c_{t}\right]$, then $c_{j} \geq c_{t}$.

(iii) The basic commutators of weight $n$ follow those of weights less than $n$. The basic commutators of weight $n$ are ordered among themselves lexicographically; that is, if $\left[b_{1}, a_{1}\right]$ and $\left[b_{2}, a_{2}\right]$ are basic commutators of weight $n$, then $\left[b_{1}, a_{1}\right]<\left[b_{2}, a_{2}\right]$ if and only if $b_{1}<b_{2}$ or $b_{1}=b_{2}$ and $a_{1}<a_{2}$.

Basic commutators are special cases of outer commutators. Outer commutators on the letters $x_{1}, x_{2}, \ldots, x_{n}, \ldots$ are defined inductively as follows:

The letter $x_{i}$ is an outer commutator word of weight one. If $u=u\left(x_{1}, \ldots, x_{s}\right)$ and $v=v\left(x_{s+1}, \ldots, x_{s+t}\right)$ are outer commutator words of weights $s$ and $t$, then $w\left(x_{1}, \ldots, x_{s+t}\right)=\left[u\left(x_{1}, \ldots, x_{s}\right), v\left(x_{s+1}, \ldots, x_{s+t}\right)\right]$ is an outer commutator word of weight $s+t$ and will be written $w=[u, v]$.

Theorem 2.2. $([3, \S 11.2])$. Let $F$ be the free group on $x_{1}, x_{2}, \ldots, x_{d}$, then for all $1 \leq i \leq n$,

$$
\frac{\gamma_{n}(F)}{\gamma_{n+i}(F)}
$$

is the free abelian group, and freely generated by the basic commutators of weights $n, n+1, \ldots, n+i-1$ on d letters.

Theorem 2.3. ([3, $\S 11.2])$. The number of basic commutators of weight $n$ on $d$ generators is given by the following formula:

$$
\chi_{n}(d)=\frac{1}{n} \sum_{m \mid n} \mu(m) d^{\frac{n}{m}},
$$


where $\mu(m)$ is the Möbius function, which is defined to be

$$
\mu(m)= \begin{cases}1 & \text { if } m=1, \\ 0 & \text { if } m=p_{1}^{\alpha_{1}} \cdots p_{k}^{\alpha_{k}} \quad \exists \alpha_{i}>1, \\ (-1)^{s} & \text { if } m=p_{1} \cdots p_{s},\end{cases}
$$

where $p_{i}$ 's are distinct prime numbers.

Let $G_{i}=\left\langle x_{i} \mid x_{i}^{k_{i}}\right\rangle$, for $i \in I$, be the cyclic group of order $k_{i}$ if $k_{i}>1$, and the infinite cyclic group if $k_{i}=0$. The $n$th nilpotent product of the family $\left\{G_{i}\right\}_{i \in I}$ is defined as follows (see $\left.[10]\right)$ :

$$
\prod_{i \in I}^{*} G_{i}=\frac{\prod_{i \in I}^{*} G_{i}}{\gamma_{n+1}\left(\prod_{i \in I}^{*} G_{i}\right)}
$$

where $\prod_{i \in I}^{*} G_{i}$ is the free product of the family $\left\{G_{i}\right\}_{i \in I}$.

Let

$$
1 \rightarrow R_{i}=\left\langle x_{i}^{k_{i}}\right\rangle \rightarrow F_{i}=\left\langle x_{i}\right\rangle \rightarrow G_{i} \rightarrow 1
$$

be a free presentation for $G_{i}$. It is routine to check that a free presentation for the $n$th nilpotent product of $\prod_{i \in I}^{*} G_{i}$ is as follows (see [9]):

$$
1 \rightarrow R=S \gamma_{n+1}(F) \rightarrow F=\prod_{i \in I}^{*} F_{i} \rightarrow \prod_{i \in I}^{*} G_{i} \rightarrow 1
$$

where $S=\left\langle x_{i}^{k_{i}} \mid i \in I\right\rangle^{F}$. Therefore, if $c \geq n$, then the $c$-nilpotent multiplier of $\prod_{i \in I}^{*} G_{i}$ is

$$
\mathcal{N}_{c} M\left(\prod_{i \in I}^{\stackrel{n}{*}} G_{i}\right)=\frac{R \cap \gamma_{c+1}(F)}{\left[R,{ }_{c} F\right]}=\frac{\gamma_{c+1}(F)}{\left[S,{ }_{c} F\right] \gamma_{c+n+1}(F)}=\frac{\gamma_{c+1}(F)}{\rho_{c+1}(S) \gamma_{c+n+1}(F)},
$$

where $\rho_{k}(S)$ is defined inductively by $\rho_{1}(S)=S$ and $\rho_{c+1}(S)=\left[\rho_{c}(S), F\right]$.

Lemma 2.4. If $1 \leq i<r$ and $(p, r)=1$ for all primes $p$ less than or equal to $i$, then $r$ divides $\left(\begin{array}{l}r \\ i\end{array}\right)$. 
Proof. Clearly $\left(\begin{array}{l}r \\ i\end{array}\right)=r\left(\frac{(r-1) \cdots(r-i+1)}{1 \times 2 \times \cdots \times i}\right)$ is an integer. For any prime $p \leq i$, $p \mid(r-1) \cdots(r-i+1)$, since $p \nmid r$. Thus, $1 \times 2 \times \cdots \times i \mid(r-1) \cdots(r-i+1)$ and, hence, the result holds.

The following consequences of the collecting process are vital in the proof of our main result.

Lemma 2.5. ([10]). Let $x, y$ be any elements of a given group and let $c_{1}, c_{2}, \ldots$ be the sequence of basic commutators of weights at least two in $x$ and $[x, y]$, in ascending order. Then

$$
\left[x^{n}, y\right]=[x, y]^{n} c_{1}^{f_{1}(n)} c_{2}^{f_{2}(n)} \cdots c_{i}^{f_{i}(n)} \cdots
$$

where

$$
f_{i}(n)=a_{1}\left(\begin{array}{c}
n \\
1
\end{array}\right)+a_{2}\left(\begin{array}{c}
n \\
2
\end{array}\right)+\cdots+a_{w_{i}}\left(\begin{array}{c}
n \\
w_{i}
\end{array}\right)
$$

with $a_{i} \in \mathbb{Z}$ and $w_{i}$ being the weight of $c_{i}$ in $x$ and $[x, y]$. If the group is nilpotent, then the expression in (2) gives an identity, and the sequence of commutators terminates.

Lemma 2.6. ([10]). Let $\alpha$ be a fixed integer and $G$ a nilpotent group of class at most $n$. If $b_{j} \in G$ and $r<n$, then

$$
\left[b_{1}, . ., b_{i-1}, b_{i}^{\alpha}, b_{i+1}, \ldots, b_{r}\right]=\left[b_{1}, \ldots, b_{r}\right]^{\alpha} c_{1}^{f_{1}(\alpha)} c_{2}^{f_{2}(\alpha)} \cdots
$$

where the $c_{k}$ 's are commutators in $b_{1}, \ldots, b_{r}$ of weight strictly greater than $r$, and every $b_{j}, 1 \leq j \leq r$ appears in each commutator $c_{k}$, the $c_{k}$ 's listed in ascending order. The $f_{i}$ 's are of the form (3), with $a_{j} \in \mathbb{Z}$ and $w_{i}=$ (the weight of $c_{i}$ on the $\left.b_{i}\right)-(r-1)$. 


\section{Main Results}

Keeping the previous notation, let $k_{i}=0$, for $1 \leq i \leq m$, and $k_{m+j}=$

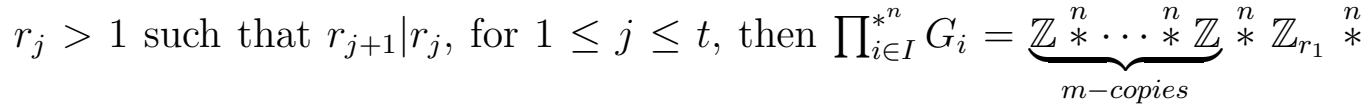
$\cdots * \mathbb{Z}_{r_{t}}$. In order to compute the $c$-nilpotent multiplier of the above group, we need two technical lemmas.

Lemma 3.1. With the above notation and assumption, if $\left(p, r_{1}\right)=1$, for all primes $p$ less than or equal to $l-i$, then $\rho_{c+i}(S) \gamma_{c+l}(F) / \rho_{c+i+1}(S) \gamma_{c+l}(F)$ is the free abelian group with a basis $D_{i, 1} \cup \cdots \cup D_{i, t}$, where

$$
\begin{array}{r}
D_{i, j}=\left\{b^{r_{j}} \rho_{c+i+1}(S) \gamma_{c+l}(F) \mid b \text { is a basic commutator of weight } c+i\right. \text { on } \\
\left.x_{1}, \ldots, x_{m}, \ldots, x_{m+j} \text { such that } x_{m+j} \text { appears in } b\right\},
\end{array}
$$

for $1 \leq i \leq l-1$ and $1 \leq j \leq t$.

Proof. Using the collecting process (see [3, §11.1]), one can easily check that $\rho_{c+i}(S) \gamma_{c+l}(F) / \rho_{c+i+1}(S) \gamma_{c+l}(F)$ is generated by all $b^{\prime} \rho_{c+i+1}(S) \gamma_{c+l}(F)$, where $b^{\prime}$ belongs to the set of basic commutators of weight $c+i, \ldots, c+l-1$ on letters $x_{1}, \cdots, x_{m}, x_{m+1}, \ldots, x_{m+t}$ such that one of the $x_{m+1}^{r_{1}}, \ldots, x_{m+t}^{r_{t}}$ appears in them. It is easy to check that all the above commutators of weight greater than $c+i$ belong to $\rho_{c+i+1}(S)$. Now, we show that if $b^{\prime}$ is one of the above commutators of weight $c+i$ such that $x_{m+j}^{r_{j}}$ appears in it, then

$$
b^{\prime} \equiv b^{r_{j}} \quad\left(\bmod \rho_{c+i+1}(S) \gamma_{c+l}(F)\right)
$$

where $b$ is a basic commutator of weight $c+i$ on $x_{1}, \ldots, x_{m}, \ldots, x_{m+t}$ such that $x_{m+j}$ appears in it. (Note that $b$ is actually a basic commutator according to the definition, and $b^{\prime}$ is the same as $b$, but the letter $x_{m+j}$ with exponent $r_{j}$.) In order to prove the above claim, first we use reverse induction on $k$ 
$(i+1 \leq k \leq l-1)$ to show that if $u$ is an outer commutator of weight $c+k$ on $x_{1}, \ldots, x_{m}, \ldots, x_{m+t}$ such that $x_{m+j}$ appears in $u$, then

$$
u^{r_{j}} \in \rho_{c+i+1}(S) \quad\left(\bmod \gamma_{c+l}(F)\right) .
$$

Let $k=l-1$ and $u=\left[\ldots, x_{m+j}, \ldots\right]$, then clearly $u^{r_{j}} \equiv\left[\ldots, x_{m+j}^{r_{j}}, \ldots\right] \in$ $\rho_{c+i+1}(S)\left(\bmod \gamma_{c+l}(F)\right)$.

Now, suppose the above property holds for every $k>k^{\prime}$. We will show that the property (5) holds for $k^{\prime}$. Let $u=\left[u_{1}, u_{2}\right]$ be an outer commutator of weight $c+k^{\prime}$ on $x_{1}, \ldots, x_{m+t}$, where $x_{m+j}$ appears in $u_{1}$. Then, by Lemma 2.5 , we have

$$
u^{r_{j}} \equiv\left[u_{1}^{r_{j}}, u_{2}\right]\left(v_{1}^{f_{1}\left(r_{j}\right)} \cdots v_{h}^{f_{h}\left(r_{j}\right)}\right)^{-1} \quad\left(\bmod \gamma_{c+l}(F)\right),
$$

where $v_{s}$ is a basic commutator of weight $w_{s}$ in $u_{1}$ and $\left[u_{1}, u_{2}\right], 1 \leq s \leq h$. Thus, $v_{s}$ is an outer commutator of weight greater than $c+k^{\prime}$ and less than $c+l$ on $x_{1}, \ldots, x_{m}, \ldots, x_{m+t}$ such that $x_{m+j}$ appears in it. By the hypothesis, since $r_{j} \mid r_{1}$ we have $\left(p, r_{j}\right)=1$ for all primes $p$ less than or equal to $l-i$. Also, it is easy to see that $w_{s} \leq(c+l)-\left(c+k^{\prime}-1\right)=l-$ $k^{\prime}+1 \leq l-i$. Therefore, by Lemma $2.4, r_{j} \mid f_{s}\left(r_{j}\right)$, and so, by induction hypothesis, $v_{s}^{f_{s}\left(r_{j}\right)} \in \rho_{c+i+1}(S)\left(\bmod \gamma_{c+l}(F)\right)$. Hence, by repeating the above process, if $u=\left[\ldots, x_{m+j}, \ldots\right]$, then $u^{r_{j}} \equiv\left[\ldots, x_{m+j}^{r_{j}}, \ldots\right] v_{1}^{\prime f_{1}^{\prime}\left(r_{j}\right)} \cdots v_{h}^{\prime f_{h}^{\prime}\left(r_{j}\right)} \in$ $\rho_{c+i+1}(S)\left(\bmod \gamma_{c+l}(F)\right)$. Now using (5), Lemma 2.6, and some commutator manipulations, the congruence (4) holds. Therefore, the set $\bigcup_{j=1}^{t} D_{i, j}$ is a generating set for $\rho_{c+i}(S) \gamma_{c+l}(F) / \rho_{c+i+1}(S) \gamma_{c+l}(F)$. On the other hand, by Theorem 2.2, distinct basic commutators are linearly independent and, hence, distinct powers of these basic commutators are also linearly independent. Therefore, the set $\bigcup_{j=1}^{t} D_{i, j}$ is a basis.

Lemma 3.2. With the notation and assumption of the previous lemma, if $\left(p, r_{1}\right)=1$ for all primes $p$ less than or equal to $l-1$, then

$$
\rho_{c+1}(S) \gamma_{c+l}(F) / \gamma_{c+l}(F)
$$


is the free abelian group with a basis $\bigcup_{i=1}^{l-1}\left(\bigcup_{j=1}^{t} D_{i, j}\right)$.

Proof. Put

$$
A_{i}=\frac{\rho_{c+i}(S) \gamma_{c+l}(F)}{\rho_{c+i+1}(S) \gamma_{c+l}(F)}, B_{i}=\frac{\rho_{c+1}(S) \gamma_{c+l}(F)}{\rho_{c+i+1}(S) \gamma_{c+l}(F)}
$$

Then, clearly the following exact sequence exists for $1 \leq i \leq l-1$

$$
0 \rightarrow A_{i} \rightarrow B_{i} \rightarrow B_{i-1} \rightarrow 0
$$

By Lemma 3.1, $B_{1}$ is a free abelian group, so the following exact sequence:

$$
0 \rightarrow A_{2} \rightarrow B_{2} \rightarrow B_{1} \rightarrow 0
$$

splits and, hence, $B_{2} \cong A_{2} \oplus B_{1}$. Also, by Lemma 3.1 every $A_{i}$ is free abelian, so by induction, every $B_{i}$ is free abelian and

$$
\frac{\rho_{c+1}(S) \gamma_{c+l}(F)}{\gamma_{c+l}(F)}=B_{l-1} \cong A_{l-1} \oplus A_{l-2} \oplus \cdots \oplus A_{2} \oplus A_{1}
$$

Now, using the basis for $A_{i}$ presented in Lemma 3.1, the result holds.

Now, we are in a position to state and prove the first main result of the paper.

Theorem 3.3. Let $G=\underbrace{\mathbb{Z}^{n} \cdots * \mathbb{Z}^{n}}_{m-\text { copies }}{ }^{n} \mathbb{Z}_{r_{1}}{ }^{n} \cdots * \mathbb{Z}_{r_{t}}$ be the nth nilpotent product of some cyclic groups, where $r_{i+1}$ divides $r_{i}$ for $1 \leq i \leq t$. If $c \geq n$ and $\left(p, r_{1}\right)=1$ for all primes $p$ less than or equal to $n$, then the c-nilpotent multiplier of $G$ is isomorphic to

$$
\mathbb{Z}^{\left(d_{m}\right)} \oplus \mathbb{Z}_{r_{1}}^{\left(d_{m+1}-d_{m}\right)} \oplus \cdots \oplus \mathbb{Z}_{r_{t}}^{\left(d_{m+t}-d_{m+t-1}\right)}
$$

where $d_{m}=\sum_{i=1}^{n} \chi_{c+i}(m)$ and $\mathbb{Z}_{r_{i}}^{(d)}$ denotes the direct sum of $d$ copies of the cyclic group $\mathbb{Z}_{r_{i}}$. 
Proof. Using the previous notation and assumption, we have

$$
\mathcal{N}_{c} M(G)=\frac{\gamma_{c+1}(F)}{\rho_{c+1}(S) \gamma_{c+n+1}(F)} \cong \frac{\gamma_{c+1}(F) / \gamma_{c+n+1}(F)}{\rho_{c+1}(S) \gamma_{c+n+l}(F) / \gamma_{c+n+1}(F)}
$$

Also, by Theorem 2.2, $\gamma_{c+1}(F) / \gamma_{c+n+1}(F)$ is a free abelian group with the basis consisting of all basic commutators of weight $c+1, \ldots, c+n$ on the letters $x_{1}, \ldots, x_{m+t}$.

Now, by considering the basis presented for $\rho_{c+1}(S) \gamma_{c+n+l}(F) / \gamma_{c+n+1}(F)$ in Lemma 3.2 and note the points that $D_{i, j}$ 's are mutually disjoint and the number of elements of $D_{i, j}$ is equal to $\chi_{c+i}(m+j)-\chi_{c+i}(m+j-1)$, the result holds.

Now the second main result of the paper, which is in turn an extension of the first one, is as follows:

Theorem 3.4. Let $G=\underbrace{\mathbb{Z}_{*}^{n} \cdots * \mathbb{Z}^{n}}_{m-\text { copies }} *^{n} \mathbb{Z}_{r_{1}}{ }^{n} \cdots * * \mathbb{Z}_{r_{t}}$ be the nth nilpotent product of some cyclic groups, where $r_{i+1}$ divides $r_{i}$, for $1 \leq i \leq t$. If $\left(p, r_{1}\right)=1$ for all primes $p$ less than or equal to $n$, then the polynilpotent multiplier with class row $c_{1}, c_{2}, \ldots, c_{s}$ of $G$ is

$$
\mathcal{N}_{c_{1}, c_{2}, \ldots, c_{s}} M(G)=\mathbb{Z}^{\left(d_{m}\right)} \oplus \mathbb{Z}_{r_{1}}^{\left(d_{m+1}-d_{m}\right)} \oplus \cdots \oplus \mathbb{Z}_{r_{t}}^{\left(d_{m+t}-d_{m+t-1}\right)},
$$

where $d_{i}=\chi_{c_{s}+1}\left(\cdots\left(\chi_{c_{2}+1}\left(\sum_{i=1}^{n} \chi_{c_{1}+i}(m)\right)\right) \cdots\right)$, for $c_{1} \geq n$ and $c_{2}, \ldots, c_{s} \geq$ 1 and $1 \leq i \leq t$.

Proof. Let $G$ be a nilpotent group of class $n \leq c_{1}$ with a free presentation $G=$ $F / R$. Since $\gamma_{c_{1}+1}(F) \leq \gamma_{n+1}(F) \leq R$, it gives $\mathcal{N}_{c_{1}} M(G)=\gamma_{c_{1}+1}(F) /\left[R,{ }_{c_{1}} F\right]$. Now, we can consider $\gamma_{c_{1}+1}(F) /\left[R,{ }_{c_{1}} F\right]$ as a free presentation for $\mathcal{N}_{c_{1}} M(G)$ and, hence,

$$
\mathcal{N}_{c_{2}} M\left(\mathcal{N}_{c_{1}} M(G)\right)=\frac{\gamma_{c_{2}+1}\left(\gamma_{c_{1}+1}(F)\right)}{\left[R,{ }_{c_{1}} F, c_{c_{2}} \gamma_{c_{1}+1} F\right]}
$$

Therefore, by (1) we have

$$
\mathcal{N}_{c_{1}, c_{2}} M(G)=\mathcal{N}_{c_{2}} M\left(\mathcal{N}_{c_{1}} M(G)\right)
$$


By continuing the above process, we can show that

$$
\mathcal{N}_{c_{1}, c_{2}, \ldots, c_{t}} M(G)=\mathcal{N}_{c_{t}} M\left(\cdots \mathcal{N}_{c_{2}} M\left(\mathcal{N}_{c_{1}} M(G)\right) \cdots\right)
$$

Using Theorem 3.3, $\mathcal{N}_{c_{1}} M(G)$ is a finitely generated abelian group of the following form:

$$
\begin{gathered}
\mathbb{Z}^{\left(\sum_{i=1}^{n} \chi_{c_{1}+i}(m)\right)} \oplus \mathbb{Z}_{r_{1}}^{\left(\sum_{i=1}^{n}\left(\chi_{c_{1}+i}(m+1)-\chi_{c_{1}+i}(m)\right)\right.} \oplus \\
\cdots \oplus \mathbb{Z}_{r_{t}}^{\left(\sum_{i=1}^{n}\left(\chi_{c_{1}+i}(m+t)-\chi_{c_{1}+i}(m+t-1)\right)\right.} .
\end{gathered}
$$

Now applying Theorem 3.3 with $n=1$, the result holds.

Remark 3.5. Let $G=\underbrace{\mathbb{Z}_{*}^{n} \cdots * \mathbb{Z}^{n}}_{m-\text { copies }}{ }^{n} \mathbb{Z}_{s_{1}} * \cdots * *^{n} \mathbb{Z}_{s_{t}}$ be the $n$th nilpotent product of some cyclic groups, where the $s_{i}$ are arbitrary natural numbers, for $1 \leq i \leq t$. If $c \geq n$ and $\left(p, s_{i}\right)=1$ for all primes $p$ less than or equal to $n$ and $1 \leq i \leq t$, then by a similar proof to Lemmas 3.1 and 3.2 and Theorem 3.3 , one can compute the $c$-nilpotent multiplier of $G$, but the formula is certainly more complicated than the one in Theorem 3.3. For example, if $G=\mathbb{Z}_{s_{1}}{ }^{n} \mathbb{Z}_{s_{2}} * \mathbb{Z}_{s_{3}}$, then $\mathcal{N}_{c} M(G)$ is as follows:

$$
\mathbb{Z}_{\alpha}^{\left(\sum_{i=1}^{n} \chi_{c+i}(2)\right)} \oplus \mathbb{Z}_{\beta}^{\left(\sum_{i=1}^{n} \chi_{c+i}(2)\right)} \oplus \mathbb{Z}_{\gamma}^{\left(\sum_{i=1}^{n} \chi_{c+i}(2)\right)} \oplus \mathbb{Z}_{\delta}^{\left(\sum_{i=1}^{n} \chi_{c+i}(3)-3 \sum_{i=1}^{n} \chi_{c+i}(2)\right)},
$$

where $\alpha=\left(s_{1}, s_{2}\right), \beta=\left(s_{2}, s_{3}\right), \gamma=\left(s_{1}, s_{3}\right), \delta=\left(s_{1}, s_{2}, s_{3}\right)$.

Moreover, using the proof of Theorem 3.4 and the above formula twice, we can compute the polynilpotent multiplier with class row $c_{1}, c_{2}$ of $G$ as follows:

$$
\begin{gathered}
\mathcal{N}_{c_{1}, c_{2}} M(G)=\mathbb{Z}_{\alpha}^{\left(e_{1}\right)} \oplus \mathbb{Z}_{\beta}^{\left(e_{1}\right)} \oplus \mathbb{Z}_{\gamma}^{\left(e_{1}\right)} \oplus \mathbb{Z}_{\delta}^{\left(e_{2}\right)} \oplus \mathbb{Z}_{(\alpha, \beta)}^{\left(e_{3}\right)} \oplus \mathbb{Z}_{(\alpha, \gamma)}^{\left(e_{3}\right)} \oplus \mathbb{Z}_{(\beta, \gamma)}^{\left(e_{3}\right)} \\
\oplus \mathbb{Z}_{(\alpha, \delta)}^{\left(e_{4}\right)} \oplus \mathbb{Z}_{(\beta, \delta)}^{\left(e_{4}\right)} \oplus \mathbb{Z}_{(\gamma, \delta)}^{\left(e_{4}\right)} \oplus \mathbb{Z}_{(\alpha, \beta, \gamma)}^{\left(e_{5}\right)} \oplus \mathbb{Z}_{(\alpha, \beta, \delta)}^{\left(e_{6}\right)} \oplus \mathbb{Z}_{(\beta, \gamma, \delta)}^{\left(e_{6}\right)},
\end{gathered}
$$

where

$$
e_{1}=\chi_{c_{2}+1}\left(\sum_{i=1}^{n} \chi_{c_{1}+i}(2)\right), e_{2}=\chi_{c_{2}+1}\left(\sum_{i=1}^{n} \chi_{c+i}(3)-3 \sum_{i=1}^{n} \chi_{c+i}(2)\right),
$$




$$
\begin{gathered}
e_{3}=\chi_{c_{2}+1}\left(2 \sum_{i=1}^{n} \chi_{c_{1}+i}(2)\right)-2 e_{1}, e_{4}=\chi_{c_{2}+1}\left(\sum_{i=1}^{n} \chi_{c+i}(3)-2 \sum_{i=1}^{n} \chi_{c+i}(2)\right)-e_{1}-e_{2}, \\
e_{5}=\chi_{c_{2}+1}\left(3 \sum_{i=1}^{n} \chi_{c_{1}+i}(2)\right)-3 \chi_{c_{2}+1}\left(2 \sum_{i=1}^{n} \chi_{c_{1}+i}(2)\right) \\
e_{6}=\chi_{c_{2}+1}\left(\sum_{i=1}^{n} \chi_{c+i}(3)-\sum_{i=1}^{n} \chi_{c+i}(2)\right)-\chi_{c_{2}+1}\left(2 \sum_{i=1}^{n} \chi_{c_{1}+i}(2)\right)- \\
\chi_{c_{2}+1}\left(\sum_{i=1}^{n} \chi_{c+i}(3)-2 \sum_{i=1}^{n} \chi_{c+i}(2)\right)
\end{gathered}
$$

It seems that the general formula in this case is more complicated than to write!

\section{References}

[1] G. Ellis, On groups with a finite nilpotent upper central quotient, Arch. Math. 70 (1998), 89-96.

[2] N. D. Gupta and M. R. R. Moghaddam, Higher Schur multiplicators of nilpotent dihedral groups, C. R. Math. Rep. Acad. Sci. Canada XIV 5 (1992), 225-230.

[3] M. Hall, The theory of groups. Macmillan Company, New York, 1959.

[4] N. S. Hekster, Varieties of groups and isologisms, J. Austral. Math. Soc. (Ser. A) 46 (1989), 22-60.

[5] C. R. Leedham-Green and S. Mckay, Baer-invariants, isologism, varietal laws and homology, Acta Math. 137 (1976), 99-150.

[6] B. Mashayekhy, Some notes on the Baer-invariant of a nilpotent product of groups, J. Algebra 235 (2001), 15-26. 
[7] M. R. R. Mogaddam and B. Mashayekhy, Higher Schur multiplicator of a finite abelian group, Algebra Colloq. 4 (3) (1997), 317-322.

[8] M. R. R. Moghaddam, The Baer-invariant of a direct product, Arch. Math. 33 (1979), 504-511.

[9] M. R. R. Moghaddam, B. Mashayekhy and S. Kayvanfar, The higher Schur multiplicator of certain class of groups, Southeast Asian Bull. Math. 27 (2003), 121-128.

[10] R. R. Struik, On nilpotent products of cyclic groups, Canad. J. Math. 12 (1960), 447-462.

Department of Pure Mathematics, P. O. Box 1159-91775, Ferdowsi University of Mashhad, Mashhad, Iran.

E-mail address: hokmabadi_ah@yahoo.com

Department of Pure Mathematics, Center of Excellence in Analysis on Algebraic Structures, Ferdowsi University of Mashhad, P. O. Box 1159-91775, Mashhad, Iran, and Institute for Studies in Theoretical Physics and Mathematics (IPM), Tehran, Iran.

E-mail address: bmashf@um.ac.ir

Department of Pure Mathematics, P. O. Box 1159-91775, Ferdowsi University of Mashhad, Mashhad, Iran.

E-mail address: fa36407@yahoo.com 